\title{
Conformal Organohalide Perovskites Enable Lasing on Spherical Resonators
}

\author{
Brandon R. Sutherland, Sjoerd Hoogland, Michael M. Adachi, \\ Chris T. O. Wong, and Edward H. Sargent
}

Version Post-Print/Accepted Manuscript

Citation Sutherland, B. R., Hoogland, S., Adachi, M. M., Wong, C. T. O., \& (published version) Sargent, E. H. (2014). Conformal Organohalide Perovskites enable Lasing on spherical Resonators. ACS Nano, 8(10), 10947-10952. doi:10.1021/nn504856g

Publisher's Statement This document is the Accepted Manuscript version of a Published Work that appeared in final form in ACS Nano, copyright (C) American Chemical Society after peer review and technical editing by the publisher. To access the final edited and published work see http://dx.doi.org/10.1021/nn504856g.

Always cite the published version, so the author(s) will receive recognition through services that track citation counts, e.g. Scopus. If you need to cite the page number of the TSpace version (original manuscript or accepted manuscript) because you cannot access the published version, then cite the TSpace version in addition to the published version using the permanent URI (handle) found on the record page. 


\section{Conformal Organohalide Perovskites Enable Lasing}

\section{on Spherical Resonators}

Brandon R. Sutherland", Sjoerd Hoogland", Michael M. Adachi, Chris T. O. Wong, and Edward H. Sargent*

Department of Electrical and Computer Engineering, University of Toronto, 10 King's College Road, Toronto, Ontario, M5S 3G4, Canada

Corresponding author email: ted.sargent@utoronto.ca perovskites, atomic layer deposition, whispering gallery mode lasing, $\mathrm{CH}_{3} \mathrm{NH}_{3} \mathrm{PbI}_{3}$

Conformal integration of semiconductor gain media is broadly important in on-chip optical communication technology. Here we deploy atomic layer deposition to create conformallydeposited organohalide perovskites — an attractive semiconducting gain medium —with the goal of achieving coherent light emission on spherical optical cavities. We demonstrate the high quality of perovskite gain media fabricated with this method, achieving optical gain in the nanosecond pulse regime with a threshold for amplified spontaneous emission of $65 \pm 8$

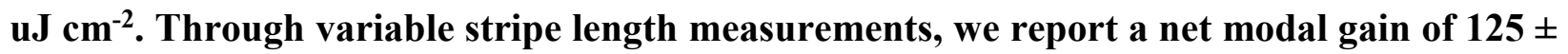
$22 \mathrm{~cm}^{-1}$ and a gain bandwidth of $50 \pm 14 \mathrm{meV}$. Leveraging the high quality of the gain medium, we conformally coat silica microspheres with perovskite to form whisperinggallery mode optical cavities and achieve lasing. 
Rapid developments in thin-film organometallic perovskites are enabling high-efficiency and low-cost solar electricity devices. ${ }^{1-9}$ The excellent photophysical properties of this material ${ }^{10}$ have been further exploited towards light-emissive technology with reports of light-emitting diodes, ${ }^{11}$ and lasing from i) bulk perovskite faceted crystals, ${ }^{12}$ ii) distributed feedback resonators, ${ }^{13}$ and iii) planar whispering gallery cavities. ${ }^{14}$

We were motivated to explore whether lasing with spherical resonators could be achieved using perovskite active layers. This would require a continuous spherical coating of the perovskite film. Spherical resonators have yielded some of the highest confinement coefficients (Q-factors) among the full suite of optical cavities, and have promising applications in on-chip integrated optical communications. ${ }^{15-17}$

Unfortunately, we found that existing methods of perovskite film deposition from the solution phase or via directional co-evaporation failed to produce, with the needed high consistency, the required conformal coverage of the curved resonator surfaces. For example, we attempted dipcoating in a liquid $\mathrm{CH}_{3} \mathrm{NH}_{3} \mathrm{PbI}_{3}$ precursor and flash anneal, but found that this approach did not produce consistent-thickness conformal layers of perovskite thin-films on the spheres, and was sensitive to drying conditions.

To couple the perovskite gain material efficiently to spherical resonators, we would require a means of forming films that would provide intimate physical and hence optical coupling, minimize scattering loss, and form a conformal and even-layer on the spheres. Techniques for conformal deposition of active materials are also important in materials science and optoelectronics broadly, such as for the direct integration of optical gain media with CMOSdefined cavities such as rib waveguides. 
We developed a perovskite growth process which utilizes atomic layer deposition (ALD), a low-temperature and low-pressure thin film formation method prized for its conformality, wide process window, and precise thickness control. We start from a film of ALD PbS, which forms the conformal seed layer. This film is then completely converted to $\mathrm{PbI}_{2}$ via exposure to $\mathrm{I}_{2}$ gas in a closed, nitrogen-inert, system. $\mathrm{PbI}_{2}$ is subsequently converted to $\mathrm{CH}_{3} \mathrm{NH}_{3} \mathrm{PbI}_{3}$ perovskite ( $\left.\mathrm{MAPbI}_{3}\right)$ through treatment with $\mathrm{CH}_{3} \mathrm{NH}_{3} \mathrm{I}$ (complete description in Methods).

\section{Results and Discussion}

Lasing relies on an active medium capable of sustaining population inversion and optical gain through stimulated emission of radiation. We studied the optical properties of the perovskite active medium to elucidate the processes that govern lasing operation. All optical studies are performed at room temperature unless otherwise noted. We began by investigating the intensitydependent spectral emission properties of a perovskite film on a lower-refractive-index substrate. We deposited a $75 \pm 18.7 \mathrm{~nm} \mathrm{MAPbI}_{3}$ thin film via perovskite ALD onto a glass slide (Figure 1a) and confirmed that the film has an absorption onset at $1.55 \mathrm{eV}$ with an absorption coefficient exceeding $10^{4} \mathrm{~cm}^{-1}$ past the bandedge (Figure $1 \mathrm{~b}$, blue), indicative of the direct bandgap of the perovskite. Under $460 \mathrm{~nm}$ continuous-wave excitation, a broad photoluminescence (PL) signal centered at $1.61 \mathrm{eV}$ with a full-width at half-maximum (FWHM) of $130 \mathrm{meV}$ (Figure 1b, red) is observed. The ultrasmall Stokes shift of $60 \mathrm{meV}$ is indicative of a sharp bandedge and minimal vibrational relaxation capable of sustaining optical gain close to the bandedge ${ }^{18}$. This increases the upper limit for the open-circuit voltage ${ }^{19}$ in perovskite photodiodes, consistent with the impressive values presented in published perovskite solar cells. 
We subsequently excited the film using a pulsed laser source with an emission wavelength of $355 \mathrm{~nm}$, pulse duration of $2 \mathrm{~ns}$, and repetition rate of $100 \mathrm{~Hz}$. Increasing the pump fluence reveals a threshold behavior at which the emission spectrum abruptly transitions from broad photoluminescence to a red-shifted narrow $17 \mathrm{meV}$ linewidth emission peak centered at $1.58 \mathrm{eV}$ (Figure 1c). The spectral narrowing for excitation above a threshold pump intensity is a signature of stimulated emission, here in the form of amplified spontaneous emission (ASE).

The resulting spectra contain superimposed emission from PL and ASE. To distinguish between these contributions, we estimate the emission intensity due to PL as twice the integrated intensity above $1.61 \mathrm{eV}$, just past the tail of the ASE signature (Figure 1d, red). The total emission from ASE is then estimated as the total integrated intensity across the entire spectral range from $1.4-1.9 \mathrm{eV}$ (Figure 1d, red + blue), minus the total PL intensity. Figure 1e shows the pump-excitation dependence of the deconvolved PL and ASE emission. The integrated power that is emitted from the broad PL portion of the spectra follows a sub-linear behavior (Figure 1e, red), indicative of state-filling near the conduction band edge. In contrast, the narrow spectral component exhibits a clear superlinear behavior with a threshold energy density of $65 \pm$ $8 \mu \mathrm{J} \mathrm{cm}^{-2}$ (Figure 1e, black). This threshold behavior with excitation intensity is another characteristic of stimulated emission, signaling that population inversion has been achieved.

The capability of perovskites to demonstrate stimulated emission in the nanosecond pulse excitation regime, orders of magnitude longer than previous reports, ${ }^{12-14}$ is a strong indication of a high-quality gain medium. The absorption coefficient at the spectral position of the ASE signal is evaluated to be $3100 \pm 830 \mathrm{~cm}^{-1}$. This value is similar to well-developed, single-crystal semiconductors such as GaAs, ${ }^{20}$ a key performance attribute that suggests promise for thin film perovskites for optical amplification. We also investigated the perovskite gain medium under 
continuous-wave, millisecond, and microsecond pulse durations, and found that emission intensity nonlinearity and spectral narrowing characteristic of amplified spontaneous emission were not observed. This is a result of the ASE threshold lying above the damage threshold of the film under these conditions. Strategies that reduce the modal losses and efficiently convey heat from the perovskite thin film could facilitate progress towards achieving population inversion under these pulse durations.

The pronounced threshold as a function of excitation intensity, from photoluminescence alone to the clear emergence of narrowband amplified spontaneous emission, indicates optical gain with a gain relaxation time that is shorter than the carrier buildup time. ${ }^{21} \mathrm{We}$ investigated the net modal optical gain in the perovskite medium using the variable stripe length characterization technique. ${ }^{22}$ Here, we excite our sample at a fixed pump fluence of $150 \mu \mathrm{J} \mathrm{cm}^{-2}$, above the threshold for ASE. We focus the circular pump beam to a stripe using a cylindrical lens. An adjustable straight-edge razor blade placed close to the sample controls the length of the excitation stripe and the emission is collected from a cleaved edge of the sample using an optical fiber (Figure 2a).

We examine the dependence of the edge-emission intensity of a stripe-pumped perovskite above the ASE threshold on the length of the excitation beam. The full spectrum for all stripe lengths is shown in Figure 2b. The emission intensity at $1.58 \mathrm{eV}$, the energy at which the ASE signal is maximum, exhibits an exponential behavior as a function of increasing stripe length prior to gain saturation. For increased stripe lengths, gain saturation occurs in regions in which free carriers have been depleted by ASE. ${ }^{23}$ An exponential fit of the collected emission in the small stripe regime (stripe length $<0.535 \mathrm{~cm}$ ) with a 1D amplifier model yields an estimated gain value of $125 \pm 22 \mathrm{~cm}^{-1}$ (Figure $2 \mathrm{~d}, \mathrm{e}$ ). This gain value corresponds to the modal gain, which 
combines the sub-unity confinement of the optical mode to the gain medium, multiplied by the material gain, and reduced by the effect of modal waveguide losses. This value for the net modal gain for 2 ns pump pulse duration is comparable to prior reports on organohalide perovskites under 150 fs pulse durations ${ }^{12}$ It is also comparable to the net modal gain in other non-singlecrystal semiconducting systems such as colloidal quantum dots,${ }^{24-27}$ silicon nanocrystals,${ }^{28}$ and semiconducting polymers. ${ }^{29}$ The spectrally-resolved modal gain bandwidth is $50 \pm 14 \mathrm{meV}$, obtained from analysis of ASE signal vs. stripe length at each wavelength. Gain bandwidths of this magnitude are similar to those found in compound semiconductors, ${ }^{20}$ consistent with the sharp bandedge of perovskites, remarkable considering the perovskite films' polycrystalline nature.

The presence of net optical gain, with a demonstration of ASE, highlights the potential for ALD perovskites to serve as active materials for lasing under nanosecond pulsed excitation. We formed the gain medium using perovskite ALD onto an optical resonator of $52 \mu \mathrm{m}$ diameter silica microspheres. Lasing can be achieved provided the cavity losses are less than the modal gain: thus only if the microspheres were conformally coated would it be possible to achieve efficient waveguiding and therefore a net positive modal gain in the film that coated the sphere.

To study the perovskite growth, we imaged the coated microspheres with scanning electron microscopy (SEM). Figure 3a shows a 45 degree tilt SEM image of the perovskite surface, revealing structured polycrystalline domains. Figures $3 b, c$ show the perovskite coating onto the curved surface of the microsphere. Using focused ion beam milling, we cut the spheres in half down the center to better study the film formation and coverage. Figures $3 \mathrm{~d}$-g reveal a uniform, continuous coating of perovskite covering the surface of the sphere with infiltration deep into the 
trenches near the sphere-substrate interface, something not readily achievable using traditional deposition methods.

We subsequently optically pumped the perovskite-coated microspheres and measured the emission spectra. We expect whispering-gallery-modes to couple into the sphere and produce optical feedback via various pathways along the circumference of the cavity, resulting in multidirectional lasing (Figure $4 a$ ). Figure $4 \mathrm{~b}$ shows the emission spectra of perovskite-coated microspheres at temperature $\mathrm{T}=80 \mathrm{~K}$ at varying excitation levels. This spectrum exhibits clearly defined modes with a regular spacing of $3 \mathrm{meV}$, corresponding to an effective refractive index of approximately 1.4 , indicating that the mode is predominantly confined to the silica-perovskite region. Figure $4 \mathrm{c}$ shows the dependence of emission intensity on pump fluence. The background signal is subtracted from the adjacent cavity mode at $1.642 \mathrm{eV}$ and the dependence of intensity on pump fluence is plotted against the background. A clear threshold is observed for the cavity mode at a pump fluence of $75 \pm 11 \mu \mathrm{J} \mathrm{cm}^{-2}$. Further, the slope-efficiency of the cavity mode signal is greater than that of the background. We attribute this combined spectral-threshold dependence to lasing cavity modes and we demonstrate that the perovskite gain medium is stable under these conditions for 24 hours (Figure 4d). The spectral bandwidth of the laser modes are dominantly limited by the resolution of the collection optics, thus we conservatively estimate the lower-bound of the Q-factor for these resonators to be of order $10^{3}$.

\section{Conclusions}

The results presented herein show that ALD perovskites offer conformal deposition onto substrates of various curvatures and aspect ratios. This fabrication strategy forms perovskite films with excellent lasing-relevant optical properties, enabling them to sustain population 
inversion when excited using low peak intensity pumping; and with excellent material properties, allowing ready formation of conformal waveguiding films when deposited on spherical resonators. Facile conformal deposition of perovskite light emitters has the potential to enable applications such as low-cost on-chip optical communication systems readily integrated with silicon photonics technology.

\section{Methods}

ALD Perovskite sample fabrication. Glass substrates were prepared by first sonicating in acetone, then isopropyl alcohol, and then deionized water, each for 15 minutes. Samples to be used for lasing studies employed silica spheres ( $52 \mu \mathrm{m}$ diameter, suspended in water) dispensed onto the surface of the glass. Excess water was dried by heating on a hotplate at $120^{\circ} \mathrm{C}$ for 1 minute. Samples were exposed to a 10 minute 100 mTorr oxygen plasma treatment immediately prior to atomic layer deposition.

$\mathrm{CH}_{3} \mathrm{NH}_{3} \mathrm{PbI}_{3}$ perovskite films are fabricated on these substrates by a three-step method. A) PbS deposition: Cleaned glass slides are placed into a Cambridge Savannah S100 atomic layer deposition system with the sample chamber held at $150{ }^{\circ} \mathrm{C}$. Alternating pulses of $\mathrm{Pb}(\mathrm{tmhd})_{2}$ and $\mathrm{H} 2 \mathrm{~S}$ precursors ( $0.5 \mathrm{~s}$ pulse duration for both) build up the $\mathrm{PbS}$ film of a desired thickness. The purge time between precursor pulses was $20 \mathrm{~s}$, and a nitrogen carrier gas was used at a volumetric flow rate of $10 \mathrm{sccm}$. B) $\mathrm{PbI}_{2}$ conversion: $100 \mathrm{mg}$ of iodine chips are placed into a $250 \mathrm{~mL}$ nitrogen-sealed container which contains the PbS films. The container is heated on a $120^{\circ} \mathrm{C}$ hotplate for 16 hours. After treatment, the samples are completely yellow and have been converted to $\mathrm{PbI}_{2}$. C) $\mathrm{MAPbI}_{3}$ conversion: The $\mathrm{PbI}_{2}$ films are dipped into a $30 \mathrm{mg} / \mathrm{mL}$ solution of 
CH3NH3I dissolved in isopropyl alcohol (IPA) for $60 \mathrm{~s}$, immediately dipped into IPA for another $60 \mathrm{~s}$, and then dried on a hot plate at $70{ }^{\circ} \mathrm{C}$ for 30 minutes.

Focused Ion Beam (FIB) and Scanning Electron Microscopy (SEM). A Hitachi table top ion mill model IM 4000 was used for sample FIB preparation. Argon ions were used at an accelerating voltage of $6 \mathrm{kV}$ and a discharge voltage of $1.5 \mathrm{kV}$. The sample was milled for 3.5 hours with the glass substrate facing the ion source to allow for gentler milling. Samples were imaged with a Hitachi NB-5000 high-resolution CFEG-SEM. An accelerating voltage of $20 \mathrm{kV}$ was used, a variable pressure of $60 \mathrm{~Pa}$, and imaged using a highly sensitive ultra-variablepressure detector.

Optical excitation and collection. Unless otherwise noted, samples were optically excited using a $355 \mathrm{~nm}$ frequency-tripled Nd:YAG laser with a pulse width of $2 \mathrm{~ns}$ and a repetition rate of $100 \mathrm{~Hz}$. All spectral data was collected using an Ocean Optics USB2000+ UV-VIS spectrometer without an input aperture, with the spectral resolution defined by the width of the input fiber core. $62.5 \mu \mathrm{m}$ core diameter fiber was used to collect all spectra, except for the data presented in Figures $4 b, c$ which was collected with a single mode fiber, with the SMF case producing a spectral resolution of $\sim 0.4 \mathrm{~nm}$, enabling us to distinguish individual laser modes. To detect WGM lasing modes, we focused the pump beam to a circular spot so that it illuminated a cluster of 4 spheres as well as some of the planar background. All measurements of laser power were taken using an Ophir Laserstar 1Z01600 single channel digital power meter.

Absorption coefficient. The optical density, $A_{O D}$, of the perovskite film was measured using a Perkin Elmer Lambda 950 spectrophotometer with an integrating sphere. A DekTak 3 profilometer was used to measure the film thickness, $d$. This was used to calculate the absorption coefficient, $\alpha=\log _{10}(\mathrm{e}) \bullet \mathrm{AoD}_{\mathrm{OD}} / \mathrm{d}$. 
Variable stripe length measurement. The variable stripe length technique was employed to determine optical gain in the $\mathrm{CH}_{3} \mathrm{NH}_{3} \mathrm{PbI}_{3}$ film. Intensity at each wavelength as a function of stripe position was fit to a linear amplifier model: $\mathrm{I}_{\mathrm{ASE}}(\mathrm{L})=\mathrm{A}\left(\mathrm{e}^{\mathrm{gL}}-1\right) / \mathrm{g}$, where $\mathrm{I}_{\mathrm{ASE}}$ is the intensity of the ASE, $\mathrm{L}$ is the stripe length, $\mathrm{g}$ is the modal gain to be determined, and $\mathrm{A}$ is a preexponential factor related to the ratio of emitted spontaneous emission to modal gain. ${ }^{23}$ 
a

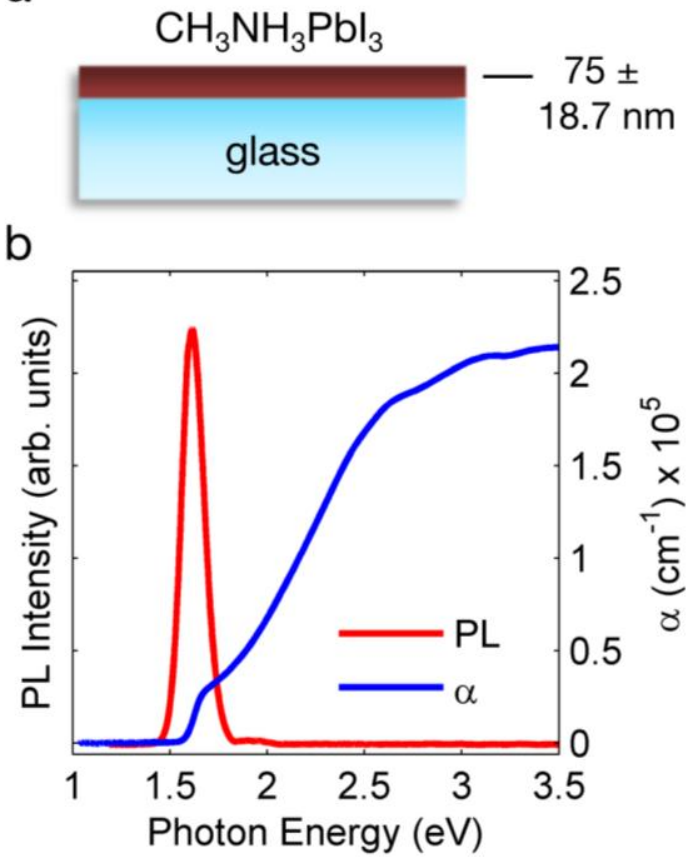

d

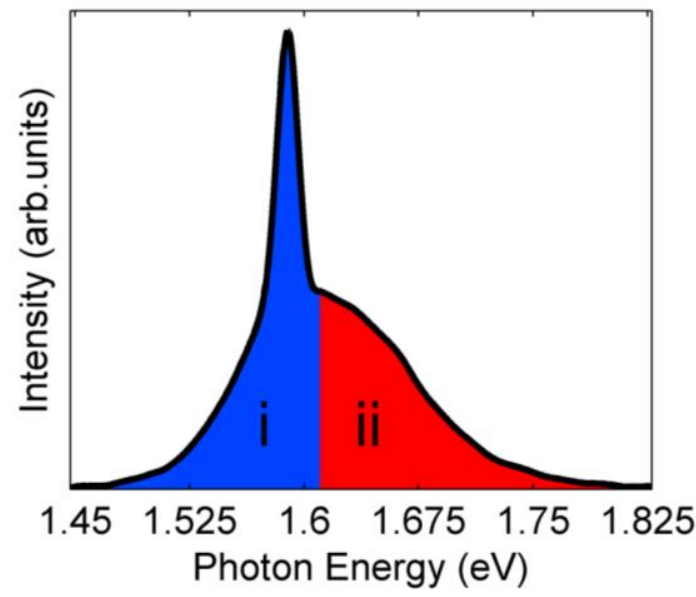

C

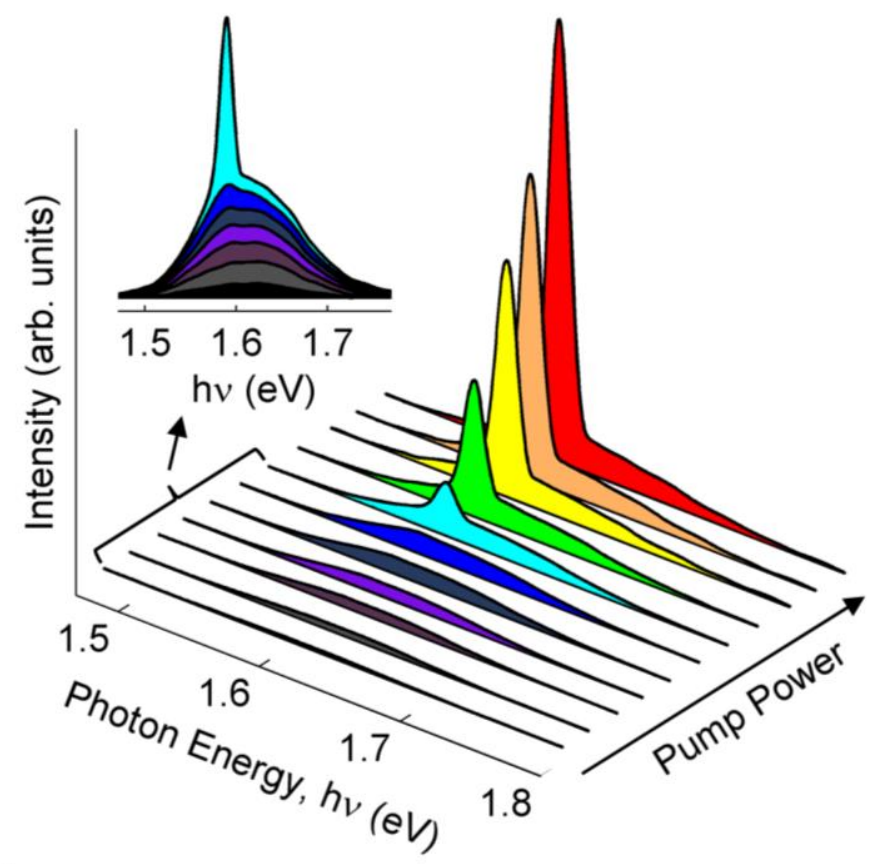

e

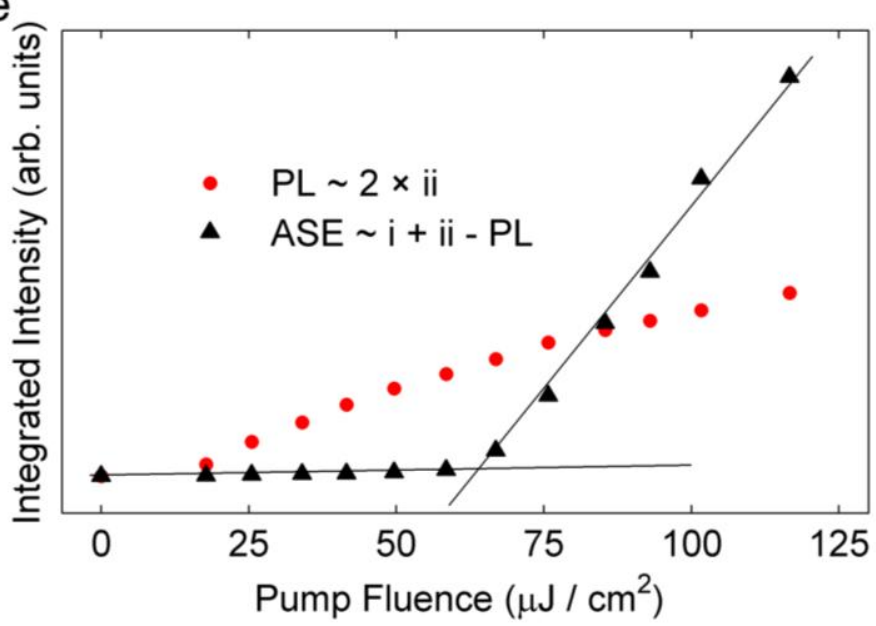

Figure 1. Amplified spontaneous emission from nanosecond pulsed excitation in $\mathrm{CH}_{3} \mathrm{NH}_{3} \mathrm{PbI}_{3}$ grown via ALD. (a) $75 \mathrm{~nm}$ of $\mathrm{MAPbI}_{3}$ is deposited onto a glass slide via perovskite-ALD. (b) Spectral absorption coefficient and photoluminescence under continuous-wave excitation. (c) Development of spectrally-narrow amplified spontaneous emission with increasing pump power. 
(d) Emission spectra at $67 \mathrm{uJ} \mathrm{cm} \mathrm{cm}^{-2}$ of ASE coupled with PL. (e) Integrated emission intensity of PL and ASE as a function of pump fluence. A clear threshold for ASE is observed at $65 \pm 8 \mathrm{uJ}$ $\mathrm{cm}^{-2}$. 

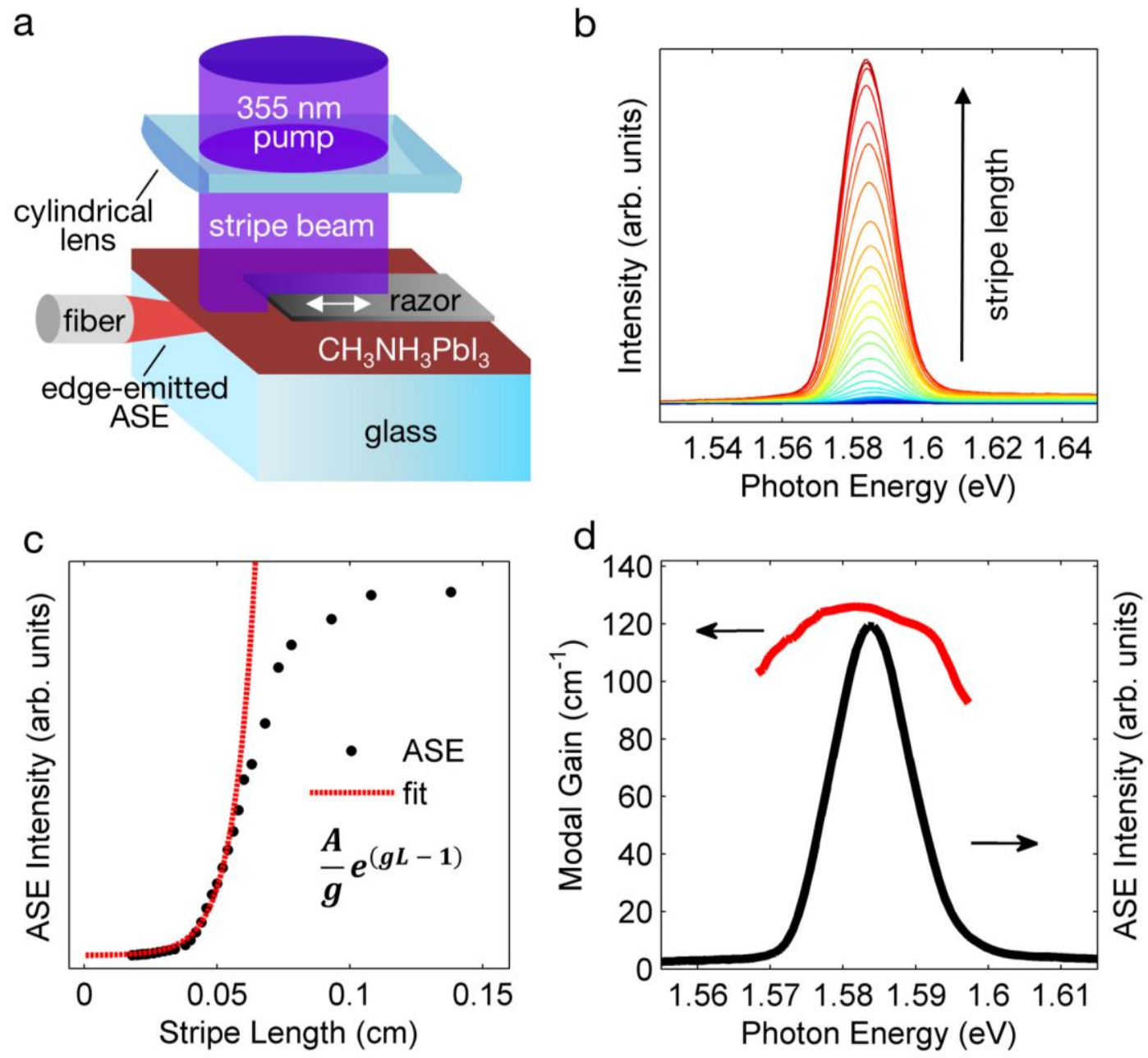

Figure 2. Net modal optical gain in $\mathrm{CH}_{3} \mathrm{NH}_{3} \mathrm{PbI}_{3}$ ALD perovskites under nanosecond pulsed excitation. (a) Variable stripe length measurement setup for gain determination. (b) Emission spectra for increasing stripe length. (c) ASE intensity at an emission photon energy of $1.58 \mathrm{eV}$ as a function of stripe length (black), and a linear amplifier fit (red) prior to the onset of gain saturation (see methods for full details). (d) Spectral dependence of modal gain (red) and sample ASE spectrum at maximum stripe length (black). The peak gain bandwidth is $125 \pm 22 \mathrm{~cm}^{-1}$ and the gain bandwidth is $50 \pm 14 \mathrm{meV}$. 

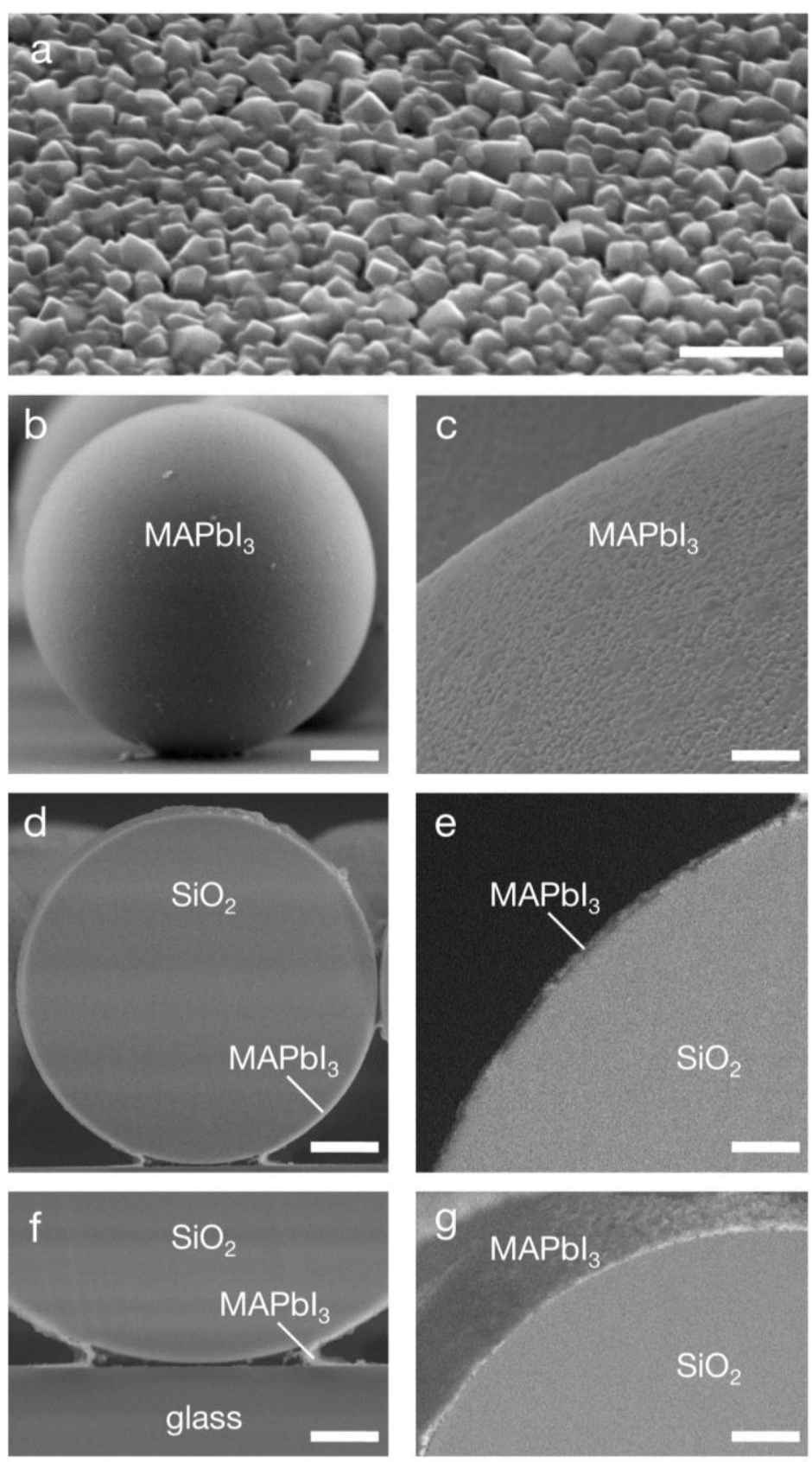

Figure 3. Scanning electron microscope images of microspheres coated via perovskite ALD. (a) 45 degree image of the perovskite surface. (b) Zoom-out of entire microsphere coated with perovskite. (c) Zoom-in showing coverage all along the curved surface of the sphere. (d) Crosssectional image of a sphere cut down the center with focused ion beam milling revealing a 
uniform coating around the circumference of the sphere. (e) Zoom-in on a cross-section of the sphere showing distinct perovskite and $\mathrm{SiO}_{2}$ regions. (f) Zoom-in of sphere-substrate interface showing a continuous film with good infiltration into the grooves. (g) Angled zoom-in of sphere cross section, showing a good uniform coating of perovskite around the surface of the sphere. Scale bars: $\mathrm{a}-\mathrm{g}: 0.2,10,2,10,3,5,4(\mu \mathrm{m})$. 
a
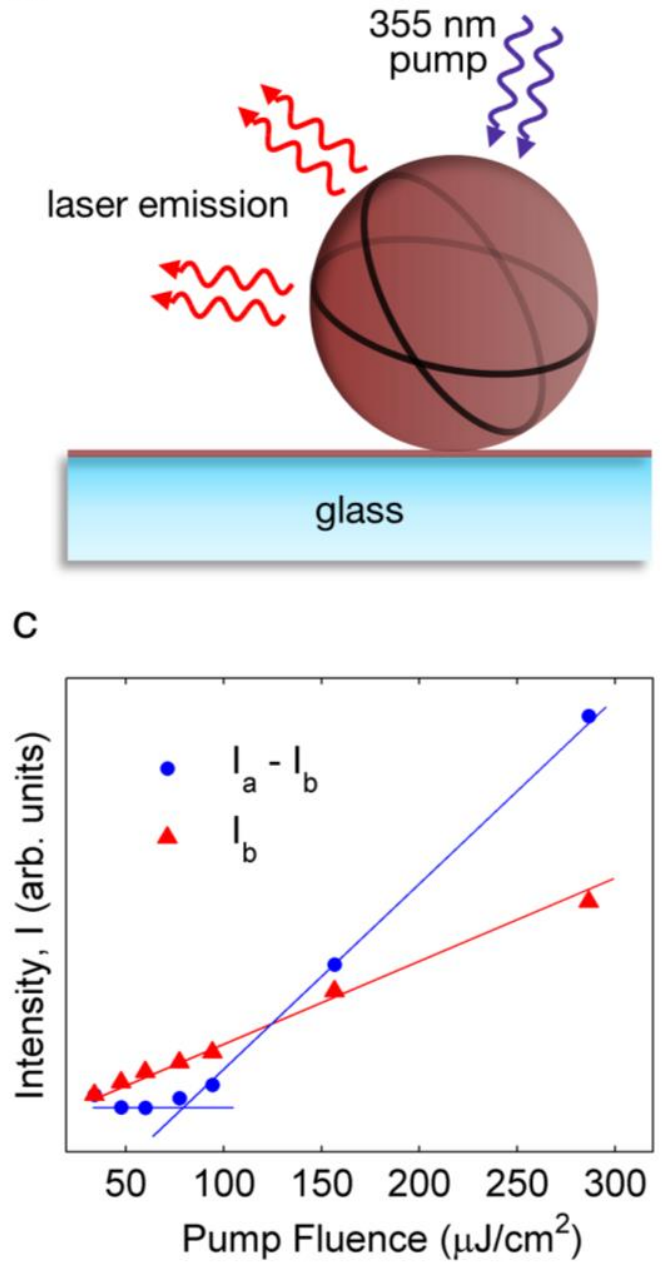

b

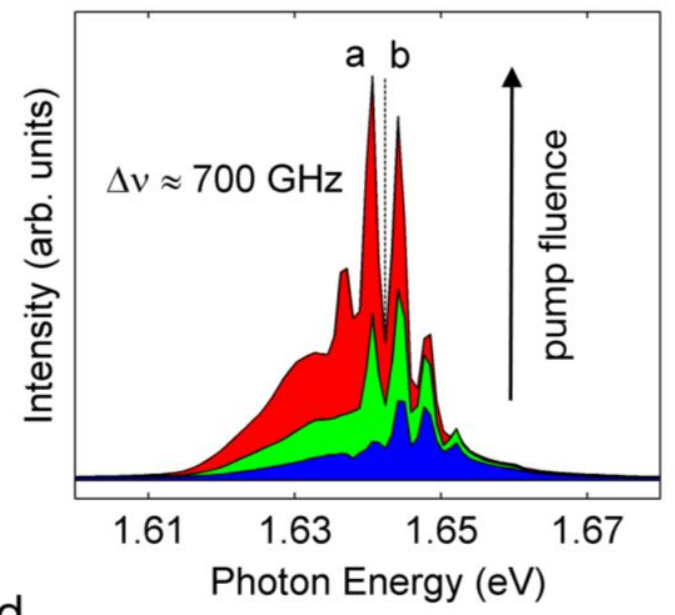

d

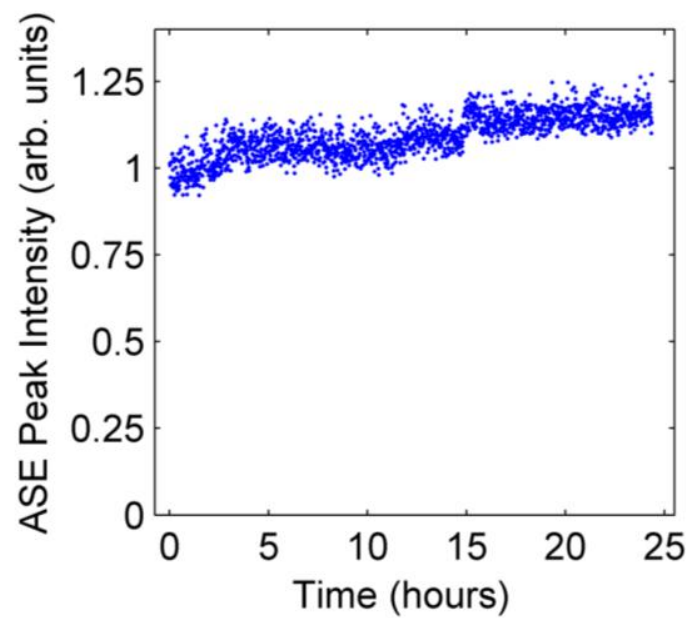

Figure 4. Modal confinement and lasing in perovskite coated silica microspheres. (a) Schematic showing a perovskite-coated microsphere absorbing pump light and coupling luminescence into the cavity, resulting in laser emission. (b) Spectral dependence of perovskite coated microsphere emission on pump power at temperature $\mathrm{T}=80 \mathrm{~K}$ for three different pump fluences (100-blue, 175-green, and 300-red $\mu \mathrm{J} \mathrm{cm}^{-2}$ ). Distinct laser modes are observed with a free spectral range of $700 \mathrm{GHz}$. (c) Lasing emission intensity dependence on pump fluence. The intensity of the dominant lasing peak at $1.642 \mathrm{eV}$, ' $\mathrm{a}$ ', with the adjacent background signal at ' $\mathrm{b}$ ' subtracted is compared to the background signal. The slope efficiency of the lasing mode (blue) is greater than 
that of the background (red), and a lasing threshold is observed at a pump fluence of $75 \pm 11 \mu \mathrm{J}$ $\mathrm{cm}^{-2}$. (d) 24 hour ASE stability under lasing conditions. 


\section{Corresponding Author}

Edward H. Sargent

ted.sargent@utoronto.ca

\section{Author Contributions}

$\uparrow$ These authors contributed equally.

\section{Acknowledgement}

This publication is based in part on work supported by an award (KUS-11-009-21) from the King Abdullah University of Science and Technology (KAUST), by the Ontario Research Fund Research Excellence Program and by the Natural Sciences and Engineering Research Council (NSERC) of Canada. The authors thank S. Boccia for FIB and SEM imaging of ALD perovskite coated microspheres, and S.-R. Jean for help with figure art. 
(1) Im, J.-H.; Lee, C.-R.; Lee, J.-W.; Park, S.-W.; Park, N.-G. 6.5\% Efficient Perovskite Quantum-Dot-Sensitized Solar Cell. Nanoscale 2011, 3, 4088.

(2) Kim, H.-S.; Lee, C.-R.; Im, J.-H.; Lee, K.-B.; Moehl, T.; Marchioro, A.; Moon, S.-J.; Humphry-Baker, R.; Yum, J.-H.; Moser, J. E.; et al. Lead Iodide Perovskite Sensitized All-Solid-State Submicron Thin Film Mesoscopic Solar Cell with Efficiency Exceeding 9\%. Sci. Rep. 2012, 2.

(3) Lee, M. M.; Teuscher, J.; Miyasaka, T.; Murakami, T. N.; Snaith, H. J. Efficient Hybrid Solar Cells Based on Meso-Superstructured Organometal Halide Perovskites. Science 2012, 338, 643-647.

(4) Noh, J. H.; Im, S. H.; Heo, J. H.; Mandal, T. N.; Seok, S. I. Chemical Management for Colorful, Efficient, and Stable Inorganic-Organic Hybrid Nanostructured Solar Cells. Nano Lett. 2013, 13, 1764-1769. Heo, J. H.; Im, S. H.; Noh, J. H.; Mandal, T. N.; Lim, C.-S.; Chang, J. A.; Lee, Y. H.; Kim, H.; Sarkar, A.; Nazeeruddin, M. K.; et al. Efficient Inorganic-organic Hybrid Heterojunction Solar Cells Containing Perovskite Compound and Polymeric Hole Conductors. Nat. Photonics 2013, 7, 486-491.

(6) Burschka, J.; Pellet, N.; Moon, S.-J.; Humphry-Baker, R.; Gao, P.; Nazeeruddin, M. K.; Grätzel, M. Sequential Deposition as a Route to High-Performance Perovskite-Sensitized Solar Cells. Nature 2013, 499, 316-319.

(7) Liu, M.; Johnston, M. B.; Snaith, H. J. Efficient Planar Heterojunction Perovskite Solar Cells by Vapour Deposition. Nature 2013, 501, 395-398.

$$
\text { Jeon, N. J.; Noh, J. H.; Kim, Y. C.; Yang, W. S.; Ryu, S.; Seok, S. I. }
$$

Solvent Engineering for High-Performance Inorganic-organic Hybrid Perovskite Solar Cells. Nat. Mater. 2014, 13, 897-903.

(9) Green, M. A.; Ho-Baillie, A.; Snaith, H. J. The Emergence of Perovskite Solar Cells. Nat. Photonics 2014, 8, 506-514. 
(10) Stoumpos, C. C.; Malliakas, C. D.; Kanatzidis, M. G. Semiconducting Tin and Lead Iodide Perovskites with Organic Cations: Phase Transitions, High Mobilities, and Near-Infrared Photoluminescent Properties. Inorg. Chem. 2013, 52, 9019-9038.

(11) Tan, Z.-K.; Moghaddam, R. S.; Lai, M. L.; Docampo, P.; Higler, R.; Deschler, F.; Price, M.; Sadhanala, A.; Pazos, L. M.; Credgington, D.; et al. Bright LightEmitting Diodes Based on Organometal Halide Perovskite. Nat. Nanotechnol. 2014.

(12) Xing, G.; Mathews, N.; Lim, S. S.; Yantara, N.; Liu, X.; Sabba, D.; Grätzel, M.; Mhaisalkar, S.; Sum, T. C. Low-Temperature Solution-Processed WavelengthTunable Perovskites for Lasing. Nat. Mater. 2014, 13, 476-480.

(13) Deschler, F.; Price, M.; Pathak, S.; Klintberg, L. E.; Jarausch, D.-D.; Higler, R.; Hüttner, S.; Leijtens, T.; Stranks, S. D.; Snaith, H. J.; et al. High Photoluminescence Efficiency and Optically Pumped Lasing in Solution-Processed Mixed Halide Perovskite Semiconductors. J. Phys. Chem. Lett. 2014, 5, 1421-1426.

(14) Zhang, Q.; Ha, S. T.; Liu, X.; Sum, T. C.; Xiong, Q. Room-Temperature Near-Infrared High-Q Perovskite Whispering-Gallery Planar Nanolasers. Nano Lett. 2014.

(15) Vernooy, D. W.; Ilchenko, V. S.; Mabuchi, H.; Streed, E. W.; Kimble, H. J. High-Q Measurements of Fused-Silica Microspheres in the near Infrared. Opt. Lett. 1998, $23,247-249$.

(16) Cai, M.; Painter, O.; Vahala, K. J. Observation of Critical Coupling in a Fiber Taper to a Silica-Microsphere Whispering-Gallery Mode System. Phys. Rev. Lett. 2000, 85, 74 .

(17) Kalkman, J.; Polman, A.; Kippenberg, T. J.; Vahala, K. J.; Brongersma, M. L. Erbium-Implanted Silica Microsphere Laser. Nucl. Instrum. Methods Phys. Res. Sect. $B$ 2006, 242, 182-185.

(18) Stranks, S. D.; Eperon, G. E.; Grancini, G.; Menelaou, C.; Alcocer, M. J. P.; Leijtens, T.; Herz, L. M.; Petrozza, A.; Snaith, H. J. Electron-Hole Diffusion Lengths 
Exceeding 1 Micrometer in an Organometal Trihalide Perovskite Absorber. Science 2013, $342,341-344$.

(19) Guyot-Sionnest, P. Electrical Transport in Colloidal Quantum Dot Films. J. Phys. Chem. Lett. 2012, 3, 1169-1175.

(20) Fermann, M.; Galvanauskas, A.; Sucha, G. Ultrafast Lasers: Technology and Applications; 1st ed.; CRC Press, 2002.

(21) Klimov, V. I. Optical Gain and Stimulated Emission in Nanocrystal Quantum Dots. Science 2000, 290, 314-317.

(22) Shaklee, K. L. Direct Determination of Optical Gain in Semiconductor Crystals. Appl. Phys. Lett. 1971, 18, 475.

(23) Negro, L. D.; Bettotti, P.; Cazzanelli, M.; Pacifici, D.; Pavesi, L. Applicability Conditions and Experimental Analysis of the Variable Stripe Length Method for Gain Measurements. Opt. Commun. 2004, 229, 337-348.

(24) Malko, A. V.; Mikhailovsky, A. A.; Petruska, M. A.; Hollingsworth, J. A.; Htoon, H.; Bawendi, M. G.; Klimov, V. I. From Amplified Spontaneous Emission to Microring Lasing Using Nanocrystal Quantum Dot Solids. Appl. Phys. Lett. 2002, 81, 1303.

(25) Petruska, M. A.; Malko, A. V.; Voyles, P. M.; Klimov, V. I. HighPerformance, Quantum Dot Nanocomposites for Nonlinear Optical and Optical Gain Applications. Adv. Mater. 2003, 15, 610-613.

(26) Dang, C.; Lee, J.; Breen, C.; Steckel, J. S.; Coe-Sullivan, S.; Nurmikko, A. Red, Green and Blue Lasing Enabled by Single-Exciton Gain in Colloidal Quantum Dot Films. Nat. Nanotechnol. 2012, 7, 335-339.

(27) Hoogland, S.; Sukhovatkin, V.; Howard, I.; Cauchi, S.; Levina, L.; Sargent, E. H. A Solution-Processed 1.53 Mm Quantum Dot Laser with TemperatureInvariant Emission Wavelength. Opt Express 2006, 14, 3273-3281. 
(28) Pavesi, L.; Dal Negro, L.; Mazzoleni, C.; Franzo, G.; Priolo, F. Optical Gain in Silicon Nanocrystals. Nature 2000, 408, 440-444.

(29) Laquai, F.; Mishra, A. K.; Müllen, K.; Friend, R. H. Amplified Spontaneous Emission of Poly(ladder-Type Phenylene)s - The Influence of Photophysical Properties on ASE Thresholds. Adv. Funct. Mater. 2008, 18, 3265-3275. 
Table of contents figure:

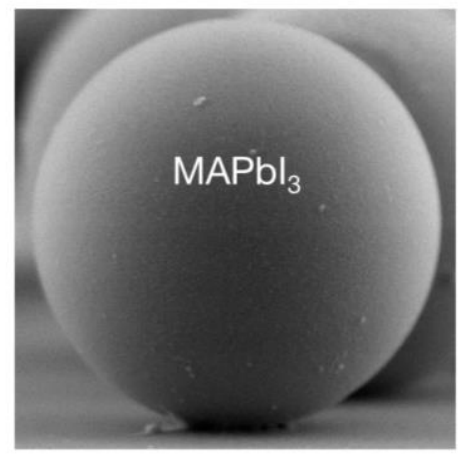

